\section{Comparación por sexo en imagen corporal, síntomas psicopatológicos y conductas alimentarias en jóvenes entre 14 y 25 años}

\author{
CLAUDIA CRUZAT-MANDICH ${ }^{\mathrm{a}}$, FERNANDA DÍAZ-CASTRILLÓN \\ PAULA LIZANA-CALDERÓN ${ }^{c}$, ANDREA CASTRO $^{d}$
}

\section{Body image, psychological symptoms and eating disorders among Chilean adolescents and young adults}

Background: The construction of body image is crucial during adolescent development. Several studies show that body dissatisfaction is common, especially among women. This is a risk factor for eating behavior disorders. Aim: To describe psychological variables and dimensions about body image among adolescents and young adults. Material and Methods: Three self-administered questionnaires, MBSRQ (Multidimensional Body Self Relations Questionnaire) that measures body image, Symptom Checklist (SCL-90) that measures the presence of psychological and psychiatric symptoms and the Eating Disorder Inventory (EDI-2), which measures eating problems, were applied to 1,438 students aged $19 \pm 2.7$ years (53\% women) from three Chilean regions. Results: Sixty five percent of respondents would like to weigh less. Compared with men, women have greater psychological distress, concerns about their appearance and their weight, are more obsessed with thinness, and have fewer behaviors aimed at solving these problems. Conclusions: A high percentage of respondents want to lose weight. In addition, women have serious desires and search for thinness.

(Rev Med Chile 2016; 144: 743-750)

Key words: Adolescent; Body Image; Feeding and Eating Disorders.

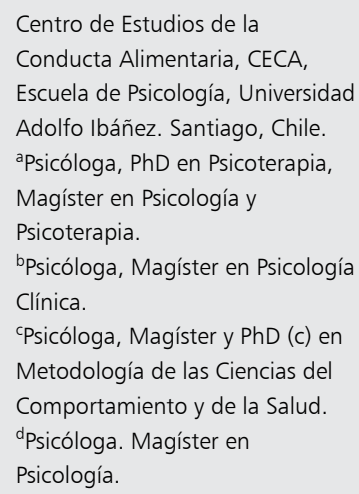

Fuente de apoyo financiero Proyecto FONDECYT regular 2014, No 1140085. Cambios en la imagen corporal en adolescentes sometidos a cirugía bariátrica.

Recibido el 17 de agosto de 2015, aceptado el 7 de mayo de 2016.

Correspondencia a:

Claudia Cruzat-Mandich

Diagonal Las Torres 2640.

Peñalolén. Santiago, Chile.

Teléfono: +562 23311648 / Fax:

56-2- 2784413

claudia.cruzat@uai.cl
L a imagen corporal es un constructo multidimensional que implica las percepciones, pensamientos y sentimientos de una persona acerca de su propio cuerpo, generalmente conceptualizado por la incorporación de la estimación del tamaño corporal, la evaluación de su atractivo y emociones asociadas a la forma y estructura corporal $^{1}$. En la conformación de la imagen corporal y, por tanto, también en la alteración de su percepción, intervienen variados aspectos biopsicosociales. Uno de los aspectos fundamentales en el desarrollo de los estereotipos corporales son los factores socioculturales, entre los cuales destacan el modelo cultural de belleza de nuestra sociedad occidental contemporánea, los patrones estéticos, la clase social, el mundo del modelaje, los medios de comunicación, la publicidad y el culto narcisista al cuerpo ${ }^{2}$. Por otro lado, entre los factores individuales que pueden influir en la apreciación de la imagen corporal, se encuentran las diferencias de género en torno a las actitudes corporales, la feminidad, el factor étnico, los patrones de crianza de la familia, la interrelación con las amistades, las críticas o burlas, el desarrollo físico y el reforzamiento social, la adolescencia, el perfeccionismo y los sitios de internet ${ }^{2}$. 
La adolescencia es una etapa crucial para el desarrollo de la imagen corporal. La cultura global presiona a las adolescentes con el mensaje de que se les valora y juzga por su cuerpo, instándoseles a compararse con el ideal estético corporal femenino de delgadez prácticamente inalcanzable. Asimismo, la presión social por la estética femenina y las transformaciones biológicas propias de ese período favorecen la mayor atención por su cuerpo en las adolescentes, y mayores niveles de insatisfacción con la imagen corporal ${ }^{2,3}$.

Además, los vínculos y la necesidad de aceptación por los pares, la admiración de figuras de culto e ídolos, más la presión de los padres, van construyendo la imagen que la joven adquiere de sí misma ${ }^{2,3}$. Por lo anterior, las adolescentes y jóvenes cursan un período altamente vulnerable para insatisfacción con la imagen corporal ${ }^{2,3}$.

La alteración de la imagen corporal ha sido considerada entre los criterios diagnósticos de los trastornos de la conducta alimentaria ${ }^{4}$. La distorsión de la imagen corporal puede oscilar desde un grado leve a severo y posee un valor predictor del pronóstico ${ }^{2}$. En Chile, se encontró que la insatisfacción corporal está presente en grado extremo en las pacientes con anorexia nerviosa (AN), bulimia nerviosa (BN) y trastorno alimentario no especificado. Las personas con anorexia mostraron mayor insatisfacción corporal acompañada de sentimientos de inutilidad personal y perfeccionismo ${ }^{5}$. También se ha planteado que las jóvenes que exhiben algún grado de insatisfacción corporal tienen alto riesgo de padecer un trastorno de la conducta alimentaria ${ }^{5-7}$.

Por otro lado, la insatisfacción corporal se ha relacionado con la presencia de obesidad en adolescentes $^{8}$. Un trabajo con estudiantes chilenos de enseñanza media mostró que los adolescentes asocian el concepto de obesidad con calificativos que revelan insatisfacción corporal, además de asociarla a depresión, ansiedad y baja autoestima, entre otros 9 . Otros estudios han encontrado correlación positiva y significativa entre índice de masa corporal (IMC) e insatisfacción con la imagen corporal ${ }^{10,11}$. En adolescentes con sobrepeso y obesidad se ha observado que las mujeres están significativamente más insatisfechas con su imagen corporal que los hombres ${ }^{10}$. Además, las mujeres sobreestiman más que los varones el IMC autopercibido/real ${ }^{12}$.

También existe evidencia que demuestra que la insatisfacción corporal puede conducir a conductas de riesgo para la salud, como lo son las dietas severas, el ejercicio extremo y el uso de sustancias para tratar de modificar la imagen corporal ${ }^{13}$.

Además, es importante destacar que la insatisfacción corporal se ha relacionado con una serie de alteraciones psicológicas, entre las que se incluyen la baja autoestima, la depresión, la ansiedad y la ideación suicida $^{14-16}$.

El número de investigaciones sobre la insatisfacción corporal y los factores asociados en muestras latinoamericanas es aún insuficiente, por ello es necesario recabar más información relevante, para la detección temprana de los trastornos o síntomas vinculados a dicha alteración.

El objetivo de este estudio es comparar por sexo, en muestra no clínica de adolescentes y jóvenes, características en relación a síntomas psicopatológicos, conductas alimentarias y dimensiones de la imagen corporal.

\section{Material y Método}

Se efectuó una investigación cuantitativa, no experimental, transversal, descriptiva y correlacional. El estudio se realizó en una muestra intencionada que alcanzó un total de 1.438 estudiantes de 14 a 25 años de edad de establecimientos educacionales de enseñanza media municipalizados, particulares subvencionados y particulares; de universidades e institutos profesionales de las regiones Metropolitana, V y VIII del país, durante el año 2014.

Se aplicaron los siguientes instrumentos:

1. Multidimensional Body-SelfRelations Questionnaire $(M B S R Q)^{17}$. Cuestionario autoaplicado de 69 ítems, que se contesta en escala de Likert de 5 puntos, según nivel de acuerdo/desacuerdo. Su versión en español fue validada por Botella, Rivas y Benito (2009) ${ }^{18}$, obteniéndose índices de consistencia interna superiores a 0,7 para todas las subescalas. Evalúa diversos aspectos de la imagen corporal.

2. Inventario de sintomas de Derogatis revisado $(S C L-90 R)^{19}$, versión validada en estudiantes universitarios chilenos ${ }^{20}$. Es un cuestionario de síntomas autoadministrado de buenas cualidades psicométricas ${ }^{20}$. El SCL-90-R se compone de 90 ítems que caracterizan al evaluado en nueve dimensiones primarias. Además, reporta tres índices globales. 
3. Eating Disorder Inventory-2 (EDI-2), validado en población chilena ${ }^{21}$, mide síntomas psicológicos asociados comúnmente a $\mathrm{AN}, \mathrm{BN}$ y otros trastornos alimentarios. La versión usada consta de 91 ítemes, con 11 subescalas. Es un cuestionario autoadministrado con seis alternativas de respuesta (de total acuerdo a ningún acuerdo). Tres de las subescalas miden actitudes y comportamientos referentes al comer, al peso y a la forma. Otras cinco se rela- cionan con construcciones más generales de la organización o los rasgos psicológicos clínicos relevantes en los trastornos alimentarios. Por último, hay tres subescalas adicionales.

4. Cuestionario demográfico-clínico, donde se preguntó sobre el sexo, edad, peso y estatura. Se consultó sobre el peso deseado y se relacionó con el peso real. Se indagó sobre antecedentes de sobrepeso y diabetes mellitus familiar (Tabla 1).

Tabla 1. Dimensiones y escalas de los instrumentos aplicados: MBRSQ, SCL-90 y EDI-2

\section{MBSRQ}

Satisfacción con la apariencia (AE): Satisfacción con la forma y atractivo físico de mi cuerpo

Orientación hacia la apariencia $(\mathrm{AO})$ : Preocupación por el aspecto físico, incluye lo percibido por otro

Evaluación del deporte y aptitudes físicas (FE): Evaluación de mi estado físico o capacidad física

Orientación por el deporte y aptitudes físicas (FO): Conductas orientadas a mantener la forma física

Interés por la salud (HO): Interés por cuidar de la salud y por la alimentación que me ayuda a tener un estilo de vida saludable

Evaluación de la salud (HE): Qué diagnóstico hago respecto de mi salud

Orientación a la enfermedad (IO): Preocupación por enfermar, signos hipocondríacos

Preocupación por el peso (OP): Preocupación por el peso y por hacer cosas para mantenerlo o modificarlo de ser necesario

Clasificación por el peso (SCW): Evaluación de mi peso desde mi perspectiva y desde la de otros

Satisfacción corporal (BAS): Grado de satisfacción con partes y aspectos de mi cuerpo

\section{SCL 90}

Somatización (SOM): Malestares por percepción de síntomas corporales dependientes fundamentalmente del SNA

Obsesiones (OBS): Síntomas de los cuadros obsesivos compulsivos

Sensitividad interpersonal (SI): Sentimientos de inferioridad e inadecuación

Depresión (DEP) Manifestaciones clínicas de síntomas depresivos

Ansiedad (ANS) Nerviosismo, tensión y ataques de pánico

Hostilidad (HOS): Expresión de agresión, irritabilidad, rabia y resentimiento

Ansiedad fóbica (FOB): Miedo persistente e irracional y conductas evitativas

Ideación Paranoide (PAR): Distorsiones cognitivas paranoides

Psicoticismo (PSIC): Sintomatología psicótica, alucinaciones y trastornos del pensamiento

Índice de gravedad global (IGS)

Índice de malestar positivo (PSDI)

\section{EDI-2}

Obsesión por la delgadez (DT): Impulso al adelgazamiento

Bulimia (B): Pensamientos o comportamientos de ingestión excesiva de comida

Insatisfacción con el propio cuerpo (BD): Insatisfacción con la forma y/o partes del cuerpo

Ineficacia (I): Sentimientos de incapacidad general, inseguridad, desprecio

Perfeccionismo (P): Grado en que la persona premia exageradamente sus logros personales

Desconfianza interpersonal (ID): Incapacidad para mostrar los sentimientos, desconfianza y dificultad de comunicación con los demás

Conciencia interoceptiva (IA): Grado de dificultad para interpretar las sensaciones corporales relacionadas con el hambre y la saciedad

Miedo a la madurez (MF): Deseos de volver a la seguridad de la infancia

Ascetismo (A): Tendencia a buscar virtud a través de la realización de ideales como la autodisciplina, dominio de sí mismo

Impulsividad (IR): Regulación del impulso

Inseguridad social (SI): Creencia de que las relaciones sociales son tensas, decepcionantes y de mala calidad 
La clasificación del estado nutricional se realizó mediante el cálculo del IMC de acuerdo con los criterios de la Organización Mundial de la Salud $(\mathrm{OMS})^{22}$.

El estudio fue aprobado por el Comité de Ética de la Universidad Adolfo Ibáñez, y de Conicyt. Se obtuvo la autorización de los directivos de los establecimientos educacionales. Todos los participantes firmaron un consentimiento informado ( $y$ en caso de menores de edad, también el adulto responsable). La información fue anónima y confidencial.

Los datos fueron analizados mediante el programa estadístico SPSS 20.0.Se efectuó análisis descriptivo a través de distribución de frecuencias y estadísticos descriptivos. La relación entre las variables de interés se evaluó a través de la prueba $t$ para diferencias de medias en muestras independientes, considerando el tamaño del efecto. Se estableció un nivel de significación estadístico de 0,05 .

\section{Resultados}

El 56,5\% de los participantes eran de género femenino y $43,5 \%$ de género masculino. Sus edades fluctuaron entre 14 y 25 años, con un promedio de 18,7 años (DE 2,7). El 31,8\% eran pre-adolescentes (entre 14 y 17 años), 41,2\% adolescentes (entre 18 y 20 años) y $27,1 \%$ jóvenes adultos (entre 21 y 25 años).

El IMC promedio fue de 22,5 (DE 3,2); se encontró $15,8 \%$ de sobrepeso, $2,2 \%$ de obesidad y $7 \%$ de bajo peso. El 74,9\% presentaba un estado nutricional normal.

Se observó que $65,3 \%$ deseaba pesar menos de lo que pesa, $17,9 \%$ deseaba pesar más y sólo 16,8\% estaba satisfecho con su peso (porcentaje que representa el peso deseado respecto del peso real).

Respecto de antecedentes familiares, 53,2\% tenía antecedente de sobrepeso en su familia y $46,8 \%$ antecedente de diabetes mellitus.

Los resultados del reporte de los cuestionarios se presentan en la Tabla 2. Se describen sólo las variables cuyos tamaños del efecto son medianos o grandes $(d>0,5)^{23}$.

En el MBRSQ existen diferencias significativas en la orientación por la apariencia (AO), es decir, las mujeres presentaron mayor preocupación por el aspecto físico que los hombres; también las mujeres presentaron menos conductas orientadas a mantener la forma física o desarrollar aptitudes físicas a través del deporte (FO) que los hombres. Por último, las mujeres presentaron mayor preocupación por el exceso de peso $(\mathrm{OP})$ que los hombres.

En relación al SCL-90, las mujeres presentaron significativamente mayores niveles de somatización (SOM) y depresión (DEP) que los hombres. Cabe destacar, aun cuando los tamaños del efecto son pequeños a medianos $(d<0,40)$, que las mujeres presentaron mayores síntomas ansiosos, y tendieron a describir sus síntomas de manera más intensa (PSDI).

En cuanto al EDI-2, se observa que las mujeres presentaban una mayor preocupación por la delgadez (DT) que los hombres, siendo el tamaño del efecto notoriamente mayor que para el resto de los ítemes. Finalmente, se observó que las mujeres presentaron mayor insatisfacción con su apariencia (BD) que los hombres.

\section{Discusión}

El presente trabajo tuvo por objeto comparar por sexo una muestra de adolescentes y jóvenes de tres regiones de Chile, para describir la relación que existe entre la imagen corporal y otras variables psicopatológicas en población normal. Esto es relevante dada la asociación entre la (in) satisfacción corporal y otras variables mediadoras, como la autoestima, la centración en el cuerpo, presión social por la delgadez y el humor negativo, todas relacionadas especialmente con riesgos de presentar algún tipo de $\mathrm{TA}^{24,25}$.

Hay 25\% de esta muestra que presenta algún riesgo de salud, ya sea por bajo o sobrepeso. Pero además, 53\% declara antecedentes familiares directos de sobrepeso u obesidad. Esto coincide parcialmente con los datos obtenidos en la Encuesta Nacional de Salud ${ }^{26}$, en que lo jóvenes entre 15 y 24 años presentan $11 \%$ de obesidad.

Llama la atención que los jóvenes y adolescentes de esta muestra señalen el antecedente familiar de diabetes mellitus en $46,5 \%$. Aunque es un dato declarado y no hay registro de cuántos de ellos están médicamente diagnosticados, es un porcentaje muy superior a lo que dicen las encuestas en Chile, en que la diabetes mellitus tipo 2 aparece en 9,4\% de la población ${ }^{26}$. Según 
Tabla 2. Estadísticos descriptivos y diferencias según género en escalas MBRSQ, SCL-90 y EDI-2

\begin{tabular}{|c|c|c|c|c|c|c|c|}
\hline \multirow{2}{*}{$\begin{array}{l}\text { Escala } \\
\text { Dimensiones }\end{array}$} & \multicolumn{2}{|c|}{ Mujeres } & \multicolumn{2}{|c|}{ Hombres } & \multicolumn{3}{|c|}{ Diferencias género } \\
\hline & $\mathbf{M}$ & DE & $\mathbf{M}$ & DE & $\mathbf{t}$ & $\mathbf{p}$ & d \\
\hline \multicolumn{8}{|l|}{ MBRSQ } \\
\hline $\mathrm{AE}$ & 3,16 & 0,86 & 3,44 & 0,73 & 6,62 & $p<0,001$ & 0,35 \\
\hline $\mathrm{AO}$ & 3,72 & 0,58 & 3,35 & 0,59 & $-11,62$ & $p<0,001$ & $0,64^{*}$ \\
\hline $\mathrm{FE}$ & 3,39 & 0,86 & 3,67 & 0,77 & 6,66 & $p<0,001$ & 0,35 \\
\hline FO & 3,32 & 0.83 & 3.73 & 0,75 & 9,69 & $p<0,001$ & $0,51 *$ \\
\hline $\mathrm{HE}$ & 3,5 & 0,72 & 3,71 & 0,65 & 5,78 & $p<0,001$ & 0,31 \\
\hline $\mathrm{HO}$ & 3,23 & 0,62 & 3,1 & 0,58 & $-3,83$ & $p<0,001$ & 0,2 \\
\hline 10 & 3,07 & 0,8 & 2,93 & 0,72 & $-3,41$ & $p<0,001$ & 0,18 \\
\hline BAS & 3,21 & 0,7 & 3,5 & 0,67 & 7,87 & $p<0,001$ & 0,42 \\
\hline OP & 2,98 & 0,93 & 2,4 & 0,77 & $-12,71$ & $p<0,001$ & $0,67^{*}$ \\
\hline SCW & 3,16 & 0,64 & 2,91 & 0,72 & $-6,83$ & $p<0,001$ & 0,36 \\
\hline \multicolumn{8}{|l|}{ SCL-90 } \\
\hline SOM & 1,07 & 0,7 & 0,74 & 0,61 & $-9,59$ & $p<0,001$ & 0,51 * \\
\hline OBS & 1,45 & 0,77 & 1,25 & 0,74 & $-4,99$ & $p<0,001$ & 0,26 \\
\hline SI & 1,02 & 0,74 & 0,79 & 0,64 & $-6,27$ & $p<0,001$ & 0,34 \\
\hline DEP & 1,14 & 0,77 & 0,78 & 0,61 & $-9,65$ & $p<0,001$ & 0,51 * \\
\hline ANS & 1,00 & 0,71 & 0,71 & 0,61 & $-8,13$ & $p<0,001$ & 0,43 \\
\hline HOS & 0,99 & 0,78 & 0,95 & 0,79 & $-0,97$ & $p=0,334$ & \\
\hline FOB & 0,5 & 0,57 & 0,33 & 0,47 & $-6,47$ & $p<0,001$ & 0,34 \\
\hline PAR & 0,86 & 0,74 & 0,8 & 0,73 & $-1,48$ & $p=0,138$ & \\
\hline PSIC & 0,61 & 0,6 & 0,5 & 0,57 & $-3,53$ & $p<0,001$ & 0,19 \\
\hline $\mathrm{TP}$ & 44,29 & 18,84 & 38,0 & 19,8 & $-6,14$ & $p<0,001$ & 0,32 \\
\hline IGS & 1,0 & 0,58 & 0,78 & 0,52 & $-7,56$ & $p<0,001$ & 0,4 \\
\hline PSDI & 1,93 & 0,47 & 1,76 & 0,43 & $-7,1$ & $p<0,001$ & 0,38 \\
\hline \multicolumn{8}{|l|}{ EDI-2 } \\
\hline DT & 6,44 & 6,22 & 2,52 & 3,61 & $-14,94$ & $p<0,001$ & $0,82^{*}$ \\
\hline $\mathrm{B}$ & 2,19 & 3,05 & 2,2 & 2,94 & $-0,07$ & $p=0,948$ & \\
\hline $\mathrm{BD}$ & 7,83 & 6,21 & 4,85 & 4,32 & $-10,69$ & $p<0,001$ & $0,57^{*}$ \\
\hline 1 & 3,46 & 4,3 & 2,72 & 3,31 & $-3,71$ & $p<0,001$ & 0,2 \\
\hline$P$ & 4,84 & 3,89 & 5,25 & 3,91 & 2,0 & $p=0,046$ & 0,11 \\
\hline ID & 3,5 & 3,58 & 3,35 & 3,38 & $-0,84$ & $p=0,404$ & \\
\hline IA & 4,99 & 4,82 & 3,49 & 3,71 & $-6,64$ & $p<0,001$ & 0,35 \\
\hline MF & 7,26 & 4,88 & 6,73 & 4,81 & $-2,03$ & $p=0,043$ & 0,11 \\
\hline A & 3,45 & 2,78 & 3,1 & 2,65 & $-2,4$ & $p=0,017$ & 0,13 \\
\hline IR & 3,44 & 4,54 & 3,55 & 4,27 & 0,47 & $p=0,639$ & \\
\hline $\mathrm{SI}$ & 3,65 & 3,79 & 3,63 & 3,49 & $-0,09$ & $p=0,93$ & \\
\hline
\end{tabular}

* Los tamaños del efecto considerados como significativos, fueron los moderados y grandes $(>0,5)$. 
datos nacionales ${ }^{26,27}$, esta patología aparece fundamentalmente en individuos mayores de 45 años, es más prevalente después de los 60 años de edad, y en estratos socioeconómicos más bajos. Otros estudios en Chile ${ }^{28,29}$ plantean que es necesario realizar intervenciones regulares para que las personas diagnosticadas mantengan un control de la enfermedad, en población chilena la adherencia a los tratamientos es baja, por lo que aumentan los riesgos y complicaciones de la enfermedad. Desde el punto de vista de salud pública, el porcentaje encontrado en esta muestra es alarmante, y es necesario seguir investigando.

Otro hallazgo relevante se refiere a la evaluación, percepción y actitudes en relación al peso. El $65 \%$ señala que desearían pesar menos, y las mujeres, en promedio, quieren pesar aun menos que los hombres. Esto concuerda con algunas investigaciones internacionales ${ }^{30}$, en que $70 \%$ de las adolescentes mujeres quieren estar más delgadas. Un porcentaje significativo de las mujeres adolescentes y jóvenes de esta muestra, que presenta un peso normal, desea bajar de peso. Diversos autores $^{25,31}$, describen que los adolescentes reciben presiones para estar más delgados, especialmente aquellos que además tienen baja satisfacción con su propio cuerpo. Específicamente en $\mathrm{Chile}^{32}$, se ha observado que la necesidad de estar más delgada se relaciona con la necesidad de alcanzar los estándares culturales de belleza. Junto a lo anterior, cabe considerar que en el período de la pubertad, los cambios corporales y la influencia social son aun más relevantes, siendo una etapa de alto riesgo para desarrollar conductas alimentarias alteradas ${ }^{30}$.

Al comparar la muestra por sexo, se obtiene que las mujeres presentan una gran preocupación por estar delgadas, la que es significativamente superior a la de los hombres en estas edades. Además, las mujeres, en comparación con los hombres, presentan mayor insatisfacción con su imagen corporal y una mayor preocupación por el peso. Esto concuerda con estudios internacionales ${ }^{33}$ y con otras investigaciones realizadas en Chile, las que han encontrado que las mujeres chilenas presentan mayor preocupación por su imagen y mayor conciencia de su peso que los hombres, y también que otras mujeres latinas ${ }^{34,35}$.

Dado estos resultados, es importante pensar si la preocupación por el peso, en estas edades, es un indicador desde el cual se evalúa sólo la apariencia.
Este es un indicador importante, porque por un lado, tiene una alta correlación con la presencia de $\mathrm{TA}^{2}$, y por otro, porque podría ser un elemento que permita intervenir tempranamente. Trabajar desde el constructo de apariencia corporal, en estas edades, puede significar el comprometer otros temas relacionados, como: hábitos alimentarios, síntomas afectivos negativos, autoestima; todos estos definidos, además, como factores de riesgo de problemas en la conducta alimentaria ${ }^{24,25}$. También podría favorecer estilos de vida más saludable, lo que es relevante dados los malos hábitos alimentarios presentados por los adolescentes y jóvenes en Chile $e^{26,36}$.

Se observa también, que son los hombres los que realizan actividades para desarrollar más habilidades y aptitudes físicas; aunque, las mujeres están preocupadas por su peso y su apariencia, y están insatisfechas con su imagen corporal, realizan en menor proporción actividades concretas para mejorarlas. Las encuestas chilenas ${ }^{26}$ plantean que hay entre $25-30 \%$ de baja actividad física a lo largo del país, independientemente de la edad y el sexo. Los promedios son levemente más altos en la $\mathrm{V}$ Región y levemente más bajos en la Región Metropolitana y VIII Región, por lo que estos datos parecen coincidir con lo observado. Es relevante pensar si la capacidad física de estos jóvenes está realmente disminuida, y sería importante investigar más sobre este indicador en población adolescente, dado que estos resultados pueden reflejar problemas de orden físico en estas poblaciones, sobre todo en patologías como la obesidad, que ha ido en aumento en los últimos años ${ }^{26}$.

Finalmente, las mujeres presentan numerosos síntomas somáticos, mayores síntomas ansiosos y depresivos que los hombres. Es decir, mujeres entre 14 y 25 años aparecen como población de riesgo; presentan: mayores malestares psicológicos, insatisfacción con su cuerpo, desean bajar proporcionalmente más de peso, están incluso obsesionadas por la delgadez, y no presentan conductas dirigidas a solucionar aquello que les preocupa. Esto es coincidente con otras investigaciones realizadas en Chile y otros países latinoamericanos $\mathrm{s}^{37,38}$ en cuanto a que la población femenina adolescente presenta mayor prevalencia y potencial de riesgo para trastornos afectivos, especialmente depresión y trastorno ansioso.

Una de las limitaciones de este estudio es el 
procedimiento de muestreo no probabilístico. Además, no se cuenta con el número de casos que no accedieron a participar; se observó mayor negación en los alumnos de los colegios, donde su participación dependía fundamentalmente del consentimiento de los padres, en comparación con los estudiantes de las universidades. En ambos casos, los instrumentos se aplicaron con autorización de las autoridades educacionales respectivas. Por otro lado, no se contó con las características sociodemográficas poblacionales, lo que hubiese permitido evaluar la representatividad de la muestra en esos factores.

Agradecimientos. Este estudio fue posible gracias al financiamiento de la Comisión Nacional de Investigación Científica y Tecnológica (CONICYT), por medio del Fondo Nacional de Desarrollo Científico y Tecnológico (FONDECYT-Regular $\mathrm{N}^{\circ}$ 1140085): Cambios en la imagen corporal en adolescentes sometidos a cirugía bariátrica.

\section{Referencias}

1. Grogan S. Body image health: contemporary perspectives. J Health Psychol 2006; 11 (4): 523-30.

2. Behar R. La construcción cultural del cuerpo: el paradigma de los trastornos de la conducta alimentaria. Rev Chil Neuro-Psiquiat 2010; 48 (4): 319-34.

3. Rodríguez S, Cruz S. Insatisfacción corporal en adolescentes latinoamericanas y españolas. Psicothema 2008 (1); 20: 131-7.

4. Asociación Americana de Psiquiatría. Guía de consulta de los criterios diagnósticos del DSM-5. Arlington, VA, Asociación Americana de Psiquiatría, 2013.

5. Behar R, Vargas C, Cabrera E. Insatisfacción corporal en los trastornos de la conducta alimentaria: un estudio comparativo. Rev Chil Neuro-Psiquiat 2011 (1); 49: 2636.

6. Stice E, Shaw H. Risk factors and prodromal eating pathology. J Chil Psychol Psychiatry 2010; 51: 518-25.

7. Moreno M, Ortíz G. Trastorno alimentario y su relación con la imagen corporal y la autoestima en adolescentes. Ter Psicol 2009; 27 (2): 181-90.

8. Neumark-Sztainer D, Paxton S, Hannan P, Haines J, Story M. Does body satisfaction matter? Five-year longitudinal associations between body satisfaction and health behaviors in adolescents females and males. J Adolesc Health 2006; 39: 244-51.

9. Cillipal E, Silva H, Vargas R, Martínez C. Significado de la obesidad para adolescentes de Temuco-Chile. Int Morphol 2006; 24 (2): 259-62.

10. Trejo P, Castro D, Facio A, Mollinedo F, Valdez G. Insatisfacción con la imagen corporal asociada al índice de masa corporal en adolescentes. Rev Cubana Enfermer 2010; 26 (3): 144-54.

11. Santana M, Silva R, Assis A, Raich R, Machado M, Pino E, et al. Factors associated with body image dissatisfaction among adolescents in public schools students in Salvador, Brazil. Nutr Hosp 2013; 28 (3): 747-55.

12. Sámano R, Rodríguez A, Sánchez-Jiménez B, Godínez E, Noriega A, Zelonka R, et al. Satisfacción de la imagen corporal en adolescentes y adultos mexicanos y su relación con la autopercepción corporal y el índice de masa corporal. Nutr Hosp 2015; 31 (3): 1082-8.

13. Garrusi B, Garousi S, Baneshi M. Body image and body change: predictive factors in an Iranian population. Int J Prev Med 2013; 4 (8): 940-8.

14. Stice E, Hayward C, Camero R, Killen J, Tailor C. Body imagen and eating related factors predict onset of depression in female adolescents: A longitudinal study. J Abnorm Psychol 2000; 109 (3): 438-44.

15. Paxon S, Eisenberg M, Neumark-Sztainer D. Prospective predictors of body dissatisfaction in adolescents girls and boys: a five-year longitudinal study. Dev Psychol 2006; 42 (5): 888-99.

16. Brausch A, Gutiérrez P. The role of body image and disordered eating as risk factors for depression and suicidal ideation in adolescents. Suicide and Life-Threat Behavi 2009; 39 (1): 58-71.

17. Brown T, Cash T, Mikulka P. Attitudinal body-image assessment: factor analisis of the Body Self Relations Questionnaire. J Pers Assess 1990; 50: 135-44.

18. Botella L, Rivas E, Benito J. Evaluación psicométrica de la imagen corporal: validación de la versión española del Multidimensional Body Self Relations Questionnaire (MBSRQ). Rev Argent Clin Psicol 2009; XVIII: 253-64.

19. Derogatis L. The SCL-90-R. Baltimore: Clinical Psychometric Research, 1975.

20. Gempp R, Avendaño C. Datos normativos y propiedades psicométricas del SCL-90-R en estudiantes universitarios chilenos. Ter Psicol 2008; 26 (1): 39-58.

21. Urzúa A, Castro S, Lillo A, Leal C. Evaluación de los trastornos alimentarios: propiedades psicométricas del Test EDI-2 en adolescentes escolarizados(as) de 13 a 18 años. Rev Chil Nutr 2009; 36 (4): 1063-73.

22. World Health Organization. Obesity: preventing and managing the global epidemic. Report of a WHO consultation (WHO technical report series 894). Genva: World Health Organization, 2000. 
23. Faul F, Erdfelder E, Lang AG, Buchner A. G*Power 3: A flexible statistical power analysis program for the social, behavioral, and biomedical sciences. Behav Res Methods 2007; 39 (2): 175-91.

24. Stice E. Risk and Maintenance factors for Eating Pathology: A Meta-Analytic Review. Psychological Bulletin 2002; 128 (5); 825-48.

25. Murray K, Rieger E, Byrne D. The relationship between Stress and Body Satisfaction in female and male adolescents. Stress Health: J Int Soc Invest Stress 2013; 31; 13-23.

26. Encuesta Nacional de Salud. ENS Chile 2009-2010. Ministerio de Salud, Chile. Disponible: http://web.minsal.cl/ portal/url/item/bcb03d7bc28b64d-fe0400 10165012d23. pdf (consultado el 20 de diciembre de 2015).

27. Asociación de Diabéticos de Chile. Estudio Nacional de Prevalencia de Diabetes en Chile 2006. Disponible en: http://www.adich.cl/Estudio.html (consultado el 20 de diciembre de 2015).

28. Lange I, Campos S, Urrutia M, Bustamante C, Alcayaga C, Téllez A, et al. Efecto de un modelo de apoyo telefónico en el auto-manejo y control metabólico de la Diabetes tipo-2 en un Centro de Atención primaria, Santiago, Chile. Rev Med Chile 2010; 138: 729-37.

29. Urzúa A, Cabrera C, González C, Arenas P, Guzmán M, Caqueo-Urízar A, et al. Análisis preliminares de la versión adaptada en población chilena de la escala de adherencia terapéutica en diabetes mellitus tipo 2EATDM-III. Rev Med Chile 2015; 143: 733-43.

30. Wertheim E, Paxton S. Body Image Development in adolescents girls. En Body Image. A handbook of Science, Practice, and Prevention. Eds. Cash \& Smolak 2011; pp. 76-84.

31. Mellor D, Mc Cabe M, Ricciardelli L, Merino ME.
(2008). Body dissatisfaction and body change behaviors in Chile: The rol of sociocultural factors. Body Image, 5, 205-215. Doi: 10.1016/j.bodyim.2008.01.004.

32. Caqueo-Urízar A, Ferrer-García M, Toro J, GutiérrezMaldonado J, Peñaloza C, Cuadros-Sosa Y, et al. Associations between sociocultural pressures to be thin, body distress, and eating disorders symptomatology among Chilean adolescent girls. Body Image 2011; 8: 78-81. Doi: 10.1016/j.bodyim.2010.11.004

33. Dion J, Blackburnn ME, Auclairb J, Laberge S, Gaudreault M, Vachon P, et al. Development an aetiology of body dissatisfaction in adolescent boys and girls. Int J Adolesc Youth 2015; 20 (2): 151-66.

34. Durán S, Díaz V, Record J, Cordón K, Encina C, Araya M. Diferencias entre índice de masa corporal real y el percibido en mujeres universitarias de Chile, Panamá y Guatemala. Nutr clín Diet Hosp 2014; 34 (2): 29-36.

35. Durán S, Rodríguez MP, Record J, Barra R, Olivares R, Tapia A, et al. Autopercepción de la imagen corporal en estudiantes universitarios de Chile y Panamá. Rev Chil Nutr 2013; 40 (1): 26-32.

36. Ratner R, Hernández P, Martel J, Atalah E.Calidad de la alimentación y estado nutricional en estudiantes universitarios de 11 regiones de Chile. Rev Med Chile 2012; 140: 1571-9.

37. De la Barra F, Vicente B, Saldivia S, Melipillán R. Estudio de epidemiología pisquiátrica en niños y adolescentes en Chile. Estadio actual. Rev Med Clin Condes 2012; 23 (5): 521-9.

38. González-Forteza C, Hermosillo de la Torre A, VacioMuro MA, Peralta R, Wagner F. Depresión en adolescentes. Un problema oculto para la salud pública y la práctica clínica. Bol Med Hosp Infant Mex 2015; 72 (2); 149-55. 